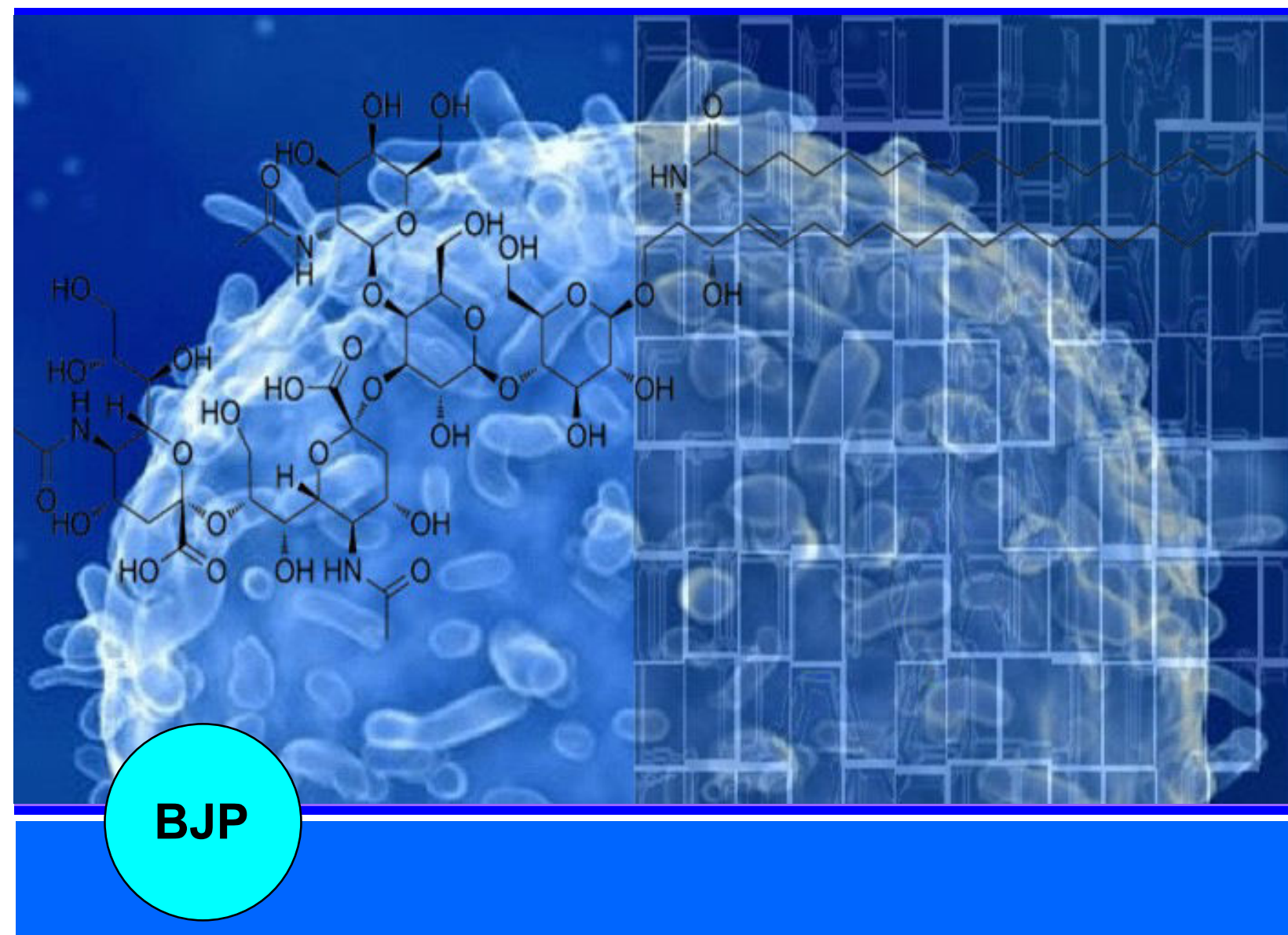

Bangladesh Journal of Pharmacology

Clinical Trial

Efficacy and safety of regorafenib versus dinutuximab with chemotherapy in Chinese children with neuroblastoma 


\title{
Efficacy and safety of regorafenib versus dinutuximab with chemotherapy in Chinese children with neuroblastoma
}

\author{
Kun Dong, Guan Wang, Zeng Liang Wang and Xueyan Wang
}

Department of Neurosurgery, Second Affiliated Hospital of Tianjin University of Traditional Chinese Medicine, Tianjin 300150, China.

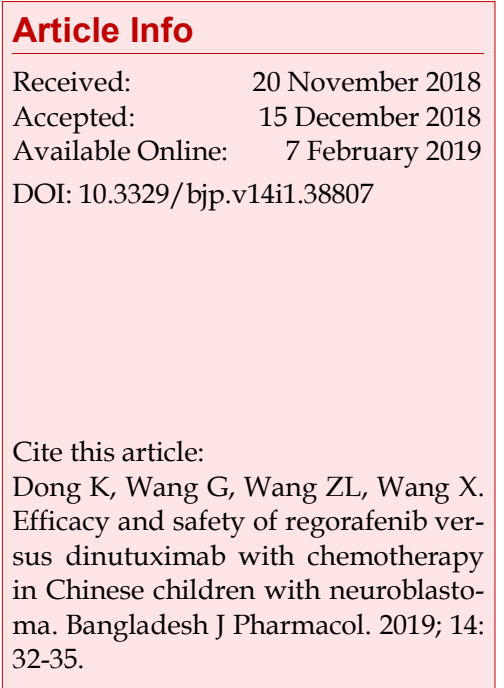

\begin{abstract}
The objective of the present study was to evaluate the efficacy and safety of regorafenib in comparison with dinutuximab with chemotherapy in Chinese children with advanced neuroblastoma. The patients aged less than 16 years who were histologically diagnosed with advanced neuroblastoma were enrolled and randomized to receive either regorafenib plus best supportive care or dinutuximab plus chemotherapy plus best supportive care in a 1:1 ratio. The tumor response assessment was made in accordance with modified international neuroblastoma response criteria. Adverse events were also assessed. Regorafenib showed prolonged overall survival and progressionfree survival than who received dinutuximab plus chemotherapy (overall survival: median 32.3 months versus 27.2 months; hazard ratio $=0.45 ; 95 \% \mathrm{CI}$ $0.11-0.13, \mathrm{p}<0.001$; progression-free survival: stratified hazard ratio $=0.48$; $95 \%$ CI $0.11-0.14 ; \mathrm{p}<0.01)$. Moreover, the overall response rate was greater in patients treated with regorafenib as compared to dinutuximab group. Regorafenib appears efficacious and has a manageable safety profile in Chinese children with advanced neuroblastoma.
\end{abstract}

\section{Introduction}

Neuroblastoma in children is one of most commonly diagnosed cancer and the leading cause of cancer mortality in the world, accounting for more than $15 \%$. The incidence and mortality of childhood neuroblastoma have increased steadily (Kushner, 2004; Boubaker and Bischof, 2003; Hiorns and Owens, 2001; Schwab et al., 2003).

The molecularly targeted therapies are being studied, and the combination of targeted agents with chemotherapy may be advantageous. Irinotecan and temozolomide can be safely administered to the patient with relapsed or refractory neuroblastoma. The monoclonal antibody as monotherapy or combination with the chemotherapy is the standard therapy for the advanced neuroblastoma. The chemotherapeutic agents common- ly used in clinical practice include irinotecan or temozolomide, and their combination regimens. In recent years, combination of temsirolimus and dinutuximab agents to chemotherapy regimens, either in the first or second line for the patient with neuroblastoma, have significantly improved overall survival, progression-free survival, and response rate (Grothey et al., 2013; Cao et al., 2016; Li et al., 2018; Xu et al., 2017). However, the majority of patients still developed resistance to the standard treatment ( $\mathrm{Xu}$ et al., 2017; Motzer et al., 2007).

Regorafenib is the first small-molecule multi-kinase inhibitor of VEGFR offers survival benefits in patient with solid cancer who has grown after first/second-line of standard therapies (Kamba and McDonald, 2007). Currently, a pharmacological study shows that regorafenib is efficacious in suppressing the tumor 
growth in pre-clinical model. However, no clinical studies have been conducted to evaluate the efficacy and safety of regorafenib in children with neuroblastoma.

Regorafenib is selective against VEGFR1, 2, and 3. It shows an acceptable safety profile and evidence of antitumor activity in patient with advanced solid tumor in several oncology clinical studies (Lacouture et al., 2008; Kamba and McDonald, 2007). In the present study, we hypothesized that regorafenib demonstrated a significant and clinically benefit in terms of overall survival in Chinese children with neuroblastoma. Efficacy of regorafenib was compared to dinutuximab.

\section{Materials and Methods \\ Study design and participants}

This is a preliminary study comparing regorafenib versus dinutuximab in Chinese children with advanced neuroblastoma. The overall safety profile of regorafenib was assessed. In patients who had Karnofsky/Lansky scores less than or equal to $50 \%$, with age less than 16 years who histologically and/or cytological were diagnosed with advanced neuroblastoma, with increased urine level of catecholamine were enrolled. The patient with insufficient response after four cycles of more than 2 chemotherapeutic agents, that included alkylating agents and platinum-containing chemotherapeutic agents were also eligible for the enrollment. Patients who had previously received any VEGFR inhibitors or had other uncontrolled medical disorders were excluded. The patients who were previously treated with anti-epidermal growth factor receptor or VEGF agents were allowed but were not mandatory.

In this study, a total of 65 patients were screened, and 60 patients were randomized to receive either regorafenib plus best supportive care $(n=30)$ or dinutuximab plus chemotherapy (irinotecan and temozolomide) plus best supportive care $(n=30)$ between January 2016 and December 2017.

\section{Study treatment}

In this study, the eligible participants were randomized to receive either regorafenib plus best supportive care or dinutuximab plus chemotherapy (irinotecan and temozolomide) plus best supportive care in a 1:1 ratio. The participants of the regorafenib group received regorafenib $160 \mathrm{mg}$ taken orally once daily for the first 21 days of each 28-day cycle. Dinutuximab (25 mg per day) was administered as intravenous infusion along with oral temozolomide (100 mg per day) and irinotecan (50 mg per day) intravenously. All randomized patients received either regorafenib plus best supportive care or dinutuximab plus chemotherapy (irinotecan and temozolomide) plus best supportive care until discontinuation due to intolerable toxicity or tumor progression or patient and investigator decision to stop the treatment or death.

\section{Efficacy and safety assessment}

Tumor response assessment was conducted every 8 weeks until the disease progression in accordance with the INR Criteria (Modified International Neuroblastoma Response Criteria), using computed tomography and/or magnetic resonance imaging evaluations to assess the overall response. The patients with partial or complete response were defined as responders. The number (percentage) of patients with partial response, complete response, stable disease and progressive disease were identified as per the standard definition. The overall survival and progression-free survival were calculated. Adverse events were recorded using CTCAE version 4.03, and recorded throughout the study period. Standard laboratory tests were performed at each visit.

\section{Statistical analysis}

Since, the present study designed as a preliminary or pilot study, thus, no official sample size calculation was performed. To assess the efficacy of treatment, progression-free survival and overall survival were compared between both the treatment groups using a log-rank test, and hazard ratio (with 95\% confidence interval) was calculated. Safety data were summarized by treatment group and presented descriptively.

\section{Results}

\section{Patient characteristics ad dose exposure}

The majority of patients in both groups had Karnofsky performance status of less than $50 \%$. In general, both groups were similar in terms of baseline demography and disease characteristics (Table I). The patients in the regorafenib group were treated for a longer period than those in the dinutuximab with chemotherapy group, with a mean treatment duration of 3.2 months versus 2.2 months, respectively. The patients in the regorafenib group received more treatment cycles than those in the dinutuximab with chemotherapy group, with a mean (SD) treatment cycle of 6.1 (1.18) versus 3.9 (2.11).

\section{Efficacy}

The patients who were treated with regorafenib showed prolonged overall survival compared to those who received dinutuximab plus chemotherapy (median 32.30 months versus 27.17 months; hazard ratio $=0.45$; 95\% CI 0.11-0.13, p<0.001). Also, progression-free survival was significantly prolonged for the patients who were treated with regorafenib compared with patients who received dinutuximab with chemotherapy (stratified hazard ratio $=0.48 ; 95 \%$ CI $0.11-0.14$; 


\begin{tabular}{|c|c|c|}
\hline \multicolumn{3}{|c|}{ Table I } \\
\hline \multicolumn{3}{|c|}{ Characteristics of the patients } \\
\hline & \multicolumn{2}{|c|}{ Best supportive care plus } \\
\hline Characteristics & Regorafenib & $\begin{array}{c}\text { Dinutuximab } \\
\text { plus chemo- } \\
\text { therapy }\end{array}$ \\
\hline \multicolumn{3}{|l|}{ Age } \\
\hline$<10$ years $(\mathrm{n})$ & 16 & 13 \\
\hline$\geq 10$ years $(n)$ & 14 & 17 \\
\hline \multicolumn{3}{|l|}{ Gender } \\
\hline Male (n) & 20 & 11 \\
\hline Female (n) & 10 & 19 \\
\hline \multicolumn{3}{|l|}{ Ethnicity } \\
\hline Han (Chinese) & 28 & 27 \\
\hline Not Han (Chinese) & 2 & 3 \\
\hline \multicolumn{3}{|c|}{ Karnofsky performance status } \\
\hline$\leq 50 \%$ & 26 & 25 \\
\hline$>50 \%$ & 4 & 5 \\
\hline \multicolumn{3}{|l|}{ Site of tumor } \\
\hline Primary & 22 & 19 \\
\hline Metastatic & 8 & 11 \\
\hline \multicolumn{3}{|c|}{ Mutations in the Kirsten ras gene status } \\
\hline Wild type & 29 & 28 \\
\hline Mutant & 1 & 2 \\
\hline $\begin{array}{l}\text { Prior use of anti-VEGF } \\
\text { treatment }\end{array}$ & 16 & 18 \\
\hline $\begin{array}{l}\text { Prior use of anti-EGFR } \\
\text { treatment }\end{array}$ & 15 & 13 \\
\hline Liver metastasis & 0 & 0 \\
\hline
\end{tabular}

$\mathrm{p}<0.001)$. Moreover, the overall response rate was greater in patients treated with regorafenib as compared to dinutuximab with chemotherapy (Table II).

\section{Safety and tolerability}

In regorafenib group, the most common treatmentrelated adverse events of all grades were hypertension, palmar-plantar erythrodysaesthesia syndrome handfoot-skin-reaction, and proteinuria (Table III). The most common treatment-related grade $\geq 3$ AESIs were hypertension, hand-foot-skin-reaction, and proteinuria.

\section{Discussion}

The overall efficacy and safety profile of regorafenib in this study was consistent with the known efficacy and safety profile of regorafenib in other cancer treatment. Moreover, the safety and efficacy profile of regorafenib was mostly similar with those of other small molecular multi-target inhibitors which also antagonizing VEGFR (Motzer et al., 2007; Escudier et al., 2007; Motzer et al., 2013; Motzer et al., 2009; Escudier et al., 2007; Kamba and McDonald, 2007; Lacouture et al., 2008). In general, regorafenib treatment was well-tolerated in Chinese children with advanced neuroblastoma. In the study, the most common treatment-related adverse event occurred in neuroblastoma patients treated with regorafenib were hypertension, hand-foot-skin-reaction, proteinuria, and hemorrhage. The most common treatment-related grade $\geq 3$ adverse events were hypertension, hand-foot-skin-reaction, and proteinuria. Hypertension was the most commonly reported treatmentrelated adverse event which is expected safety concern with various anti-VEGF/VEGFR agents (Motzer et al., 2013; Motzer et al., 2009; Escudier et al., 2007; Kamba and McDonald, 2007; Lacouture et al., 2008). This was

\section{Table II}

\section{Efficacy comparison of regorafenib vs dinutuximab plus chemotherapy}

\begin{tabular}{|c|c|c|}
\hline & \multicolumn{2}{|c|}{ Best supportive care plus } \\
\hline Variables & $\begin{array}{l}\text { Regorafenib } \\
(\mathrm{n}=30)\end{array}$ & $\begin{array}{l}\text { Dinutuximab plus chemotherapy } \\
\qquad(\mathrm{n}=30)\end{array}$ \\
\hline \multicolumn{3}{|l|}{ Overall survival } \\
\hline Median in months (95\% CI) & $32.3(8.1-10.5)$ & $27.2(5.7-8.1)$ \\
\hline Stratified hazard ratio $(95 \% \mathrm{CI}), \mathrm{p}$ value & \multicolumn{2}{|c|}{$0.45(0.11-0.13), \mathrm{p}<0.001$} \\
\hline \multicolumn{3}{|l|}{ Progression-free survival } \\
\hline Median in months $(95 \% \mathrm{CI})$ & $8.8(4.7-10.6)$ & $5.1(2.8-8.8)$ \\
\hline Stratified hazard ratio $(95 \% \mathrm{CI}), \mathrm{p}$ value & \multicolumn{2}{|c|}{$0.48(0.11-0.14), \mathrm{p}<0.001$} \\
\hline \multicolumn{3}{|l|}{ Overall response (n) } \\
\hline Complete response & 2 & 1 \\
\hline Partial response & 13 & 9 \\
\hline Stable disease & 12 & 15 \\
\hline Progressive disease & 3 & 5 \\
\hline
\end{tabular}


Table III

Comparison of treatment-related adverse events in
children with neuroblastoma (safety population)

Best supportive care plus

Adverse events Regorafenib Dinutuximab plus chemotherapy

\begin{tabular}{lll}
\hline Hypertension & 13 & 11 \\
Hand-foot skin reac- & 14 & 12
\end{tabular}

tion

$\begin{array}{lll}\text { Rash } & 12 & 9\end{array}$

$\begin{array}{lll}\text { Proteinuria } & 19 & 18\end{array}$

Occult blood positive $\quad 8 \quad 12$

$\begin{array}{lll}\text { Epistaxis } & 9 & 19\end{array}$

$\begin{array}{lll}\text { Hematuria } & 12 & 12\end{array}$

$\begin{array}{lll}\text { Hypothyroidism } & 20 & 19\end{array}$

$\mathrm{n}=30$ in each group; No Grade 5 treatment-related treatmentemergent adverse event occurred at an overall rate $\geq 5 \%$

possibly due to decrease release of nitric oxide, which is essential for maintaining blood pressure (Kamba and McDonald, 2007; Lacouture et al., 2008). Hand-foot-skin -reaction and proteinuria occurred after treatment is possibly due to the inhibition of VEGF receptors (Kamba and McDonald, 2007; Lacouture et al., 2008). No severe and fatal treatment-related toxicities have been reported in patients treated with regorafenib. How -ever, it has been observed with other small molecule VEGFR inhibitors (Lacouture et al., 2008). Overall, regorafenib appeared efficacious and have a manageable safety profile and well tolerated in the treatment population. The majority of adverse events were associated with the mechanism of action of the regorafenib, and consistent with those of its therapeutic class. Also, regorafenib demonstrated a significant and clinically benefit in terms of overall survival and progression-free survival as compared with dinutuximab with chemotherapy in Chinese children with advanced neuroblastoma.

\section{Conclusion}

Regorafenib appears efficacious and has a manageable safety profile in Chinese children with advanced neuroblastoma.

\section{Ethical Issue}

Written informed consent was taken from each patient and ethics committee approval was obtained from the Institutional Ethics Committee of Dezhou People's Hospital.

\section{References}

Boubaker A, Bischof DA. Nuclear medicine procedures and neuroblastoma in childhood: Their value in the diagnosis, staging and assessment of response to therapy. Q J Nucl Med. 2003; 47: 31-40.

Cao J, Zhang J, Peng W. A Phase I study of safety and pharmacokinetics of fruquintinib, a novel selective inhibitor of vascular endothelial growth factor receptor-1, -2 , and -3 tyrosine kinases in Chinese patients with advanced solid tumors. Cancer Chemother Pharmacol. 2016; 78: 259-69.

Escudier B, Eisen T, Stadler WM, Szczylik C, Oudard S, Siebels M, Negrier S, Chevreau C, Solska E, Desai AA, Rolland F, Demkow T, Hutson TE, Gore M, Freeman S, Schwartz B, Shan M, Simantov R, Bukowski RM; TARGET Study Group. Sorafenib in advanced clear-cell renal-cell carcinoma. N Engl J Med. 2007; 356: 125-34.

Grothey A, Van Cutsem E, Sobrero A. Regorafenib monotherapy for previously treated metastatic colorectal cancer (CORRECT): An international, multicentre, randomised, placebo-controlled, phase 3 trial. Lancet 2013; 381: 303-12.

Hiorns MP, Owens CM. Radiology of neuroblastoma in children. Eur Radiol. 2001; 11: 2071-81.

Kamba T, McDonald DM. Mechanisms of adverse effects of anti-VEGF therapy for cancer. Br J Cancer. 2007; 96: 1788-95.

Kushner BH. Neuroblastoma: A disease requiring a multitude of imaging studies. J Nucl Med. 2004; 45: 1172-88.

Lacouture ME, Reilly LM, Gerami P. Hand foot skin reaction in cancer patients treated with the multikinase inhibitors sorafenib and sunitinib. Ann Oncol. 2008; 19: 1955-61.

Li J, Qin S, Xu R. Effect of fruquintinib vs placebo on overall survival in patients with previously treated metastatic colorectal cancer The FRESCO Randomized Clinical Trial. JAMA. 2018; 319: 2486-96.

Motzer RJ, Hutson TE, Cella D. Pazopanib versus sunitinib in metastatic renal-cell carcinoma. N Engl J Med. 2013; 369: 722 -31 .

Motzer RJ, Hutson TE, Tomczak P. Overall survival and updated results for sunitinib compared with interferon alfa in patients with metastatic renal cell carcinoma. J Clin Oncol. 2009; 27: 3584-90.

Motzer RJ, Hutson TE, Tomczak P. Sunitinib versus interferon alfa in metastatic renal-cell carcinoma. N Engl J Med. 2007; 356: 115-24.

$\mathrm{Xu} \mathrm{RH}$, Jin L, Yuxian B. Safety and efficacy of fruquin-tinib in patients with previously treated metastatic colorectal cancer: A phase Ib study and a randomized double-blind phase II study. J Hematol Oncol. 2017; 10: 22.

Schwab M, Westermann F, Hero B, Berthold F. Neuroblastoma: Biology and molecular and chromosomal pathology. Lancet Oncol. 2003; 4: 472-80. 